\title{
Comparación de polvos PA12 residuo de sinterizado láser selectivo con fines de reúso funcional
}

\section{Comparison of residual PA12 powders from selective laser sintering for functional reuse}

Sergio Esteban Arbeláez-Ríos ${ }^{1}$, César Omar Balderrama-Armendáriz¹, Santos Adriana Martel-Estrada1, David Cortés-Sáenz, María Paulina Restrepo-Salgado1

1 Universidad Autónoma de Ciudad Juárez

\section{RESUMEN}

Actualmente, la poliamida 12 (PA12) es uno de los polímeros con mayor uso en la industria de la manufactura aditiva en sus diversas tecnologías. En el Sinterizado Láser Selectivo (SLS), la pureza del material permite obtener piezas de buena calidad, sin embargo, los usuarios prefieren disponerlo como desecho debido a que su integridad se degrada si se intenta el reúso. En el presente trabajo se reporta una comparación de polvos de PA12 virgen y residuo, analizando si existe algún cambio en sus características morfológicas, químicas y cristalinas. Para ello, se realizaron los análisis micrográfico por medio de SEM-EDS; estructural, de los grupos funcionales por FTIR; y cristalino, por medio de XRD. Como resultado, se obtuvo que el tamaño de partícula para la calidad residuo presenta mayores tamaños respecto al virgen. Los análisis de FTIR y XRD mostraron la presencia de los mismos grupos funcionales en la PA12 virgen y PA12 residuo, así como propiedades cristalinas similares en ambas sustancias. Se concluye con una recomendación para el reúso del polímero, considerando estrategias en cuanto al aumento de tamaño de la partícula.

PALABRAS CLAVE: Poliamida; residuos; SLS; manufactura aditiva.

\section{ABSTRACT}

Polyamide 12 (PA12) is currently one of the most used polymers in the additive manufacturing industry in its various technologies. In Selective Laser Sintering (SLS), the purity of the material allows obtaining good quality parts. However, it is not clear an appropriate reuse of the material due to degradation in its quality during the process. Hence, users prefer not to reuse it and it is arranged as waste. In this paper, a comparison of PA12 powders virgin and waste is reported, analyzing if there is any change in its morphological, chemical, and crystalline characteristics. Then, a micrographic analysis is performed using SEM-EDS, structural analysis of functional groups by FTIR, and crystalline through XRD (X-Ray Diffraction). As a result, it was obtained that the particle size for residue quality is larger than the virgin. On the other hand, FTIR and XRD analyses showed the presence of the same functional groups in virgin PA12 and residue and similar crystalline properties in both substances. It concludes with a recommendation for how to reuse the polymer considering strategies for increasing particle size.

KEYWORDS: Polyamide; residues; SLS; additive manufacturing.

Correspondencia: Sergio Esteban Arbeláez Ríos Institución: Universidad Autónoma de Ciudad Juárez / Instituto de Ingeniería y Tecnología

Dirección: Av. Del Charro núm. 450, col. Partido Romero, Ciudad Juárez, Chihuahua, México, C. P. 32310

Correo electrónico: al194449@alumnos.uacj.mx
Fecha de recepción: Marzo 24, 2020; Fecha de aceptación: Abril 4, 2021. Fecha de publicación: Abril 26, 2021.

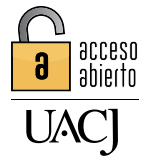

Licencia Creative Commons

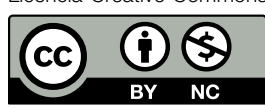




\section{INTRODUCCIÓN}

El uso de materiales poliméricos por parte de la industria en el desarrollo de productos y/o servicios ha ocasionado una gran contaminación debido a los residuos generados. Para el año 2015, únicamente la Unión Europea reportó un consumo de 49 millones de toneladas [1]. La poliamida (PA), conocida también como nylon, es un polímero cristalino producido comúnmente a partir de la condensación de un diácido y una diamina ${ }^{[2]}$ que se emplea en diversas aplicaciones gracias a sus excelentes propiedades como termoplástico de alta resistencia, su desempeño como aislante eléctrico $\mathrm{y}$ en procesos que demandan contacto con sustancias químicas, entre otras características ${ }^{[3]}$. Estos factores propician que el uso de este plástico de ingeniería sea del $14.3 \%$ [1].

Existen diversos procesos en la manufactura de la poliamida, siendo la manufactura aditiva (AM, por sus siglas en inglés) uno de los que tiene mayor auge en este momento. Diversas tecnologías de AM han sido desarrolladas para el procesamiento de polímeros, como el modelado por deposición fundida (FDM), la estereolitografía (SLA) y la manufactura de objetos laminados (LOM), entre otras [4]. Una de estas tecnologías, empleada para producir partes por medio del sinterizado selectivo $y$ desarrollada a partir de la década de los ochenta por Carl R. Deckard y otros miembros de la Universidad de Austin [5], fue adquirida en 2001 por 3D Systems y desde entonces fue registrada y conocida como sinterizado láser selectivo (SLS) [6], [7].

El SLS, que se encuentra incluido dentro de los procesos conocidos con el nombre de fusión de cama de polvo o PBF (Powder Bed Fusion), puede usar como materia prima materiales poliméricos, metálicos y combinaciones metal-metal, metal-polímero y metal-cerámico en presentación de polvos [8], [9], [10].

En su proceso, el material es depositado en la cámara de construcción en forma de lecho o cama de polvo y es sinterizado de forma localizada en un sistema coordenado 2D (plano XY) por un láser. Una vez que finaliza el sinterizado de la capa, el pistón de la cámara de construcción baja y una nueva capa es depositada, lo cual se repite hasta que la pieza es fabricada por completo [11], ${ }^{[12]}$ y es conservada en el lecho a una temperatura específica mientras esta se enfría lentamente.
Hay una gran variedad de empresas industriales dedicadas a la investigación y fabricación de este tipo de tecnología y otras relacionadas con la manufactura aditiva, p. ej., 3DSystems, Sintratec, EOS, etc., que brindan soluciones tecnológicas para procesos de impresión por SLS [13], [14], [15] gracias al desarrollo de máquinas como la ProX SLS 6100 de 3DSystems, Sintratec S2 Starter y la EOS Formiga P110 Velocis, que permiten el proceso SLS de polvos poliméricos como el nylon, posibilitando así el desarrollo de aplicaciones mayormente para el prototipado de productos. Sin embargo, también se usan en el campo de la manufactura de productos finales tecnológicos, industriales, aeronáuticos, automovilísticos y de medicina, entre otros. Algunos ejemplos de esto se pueden apreciar en sistemas de compresión de aire, hidráulicos y de combustible o de dispositivos médicos ${ }^{[3]}$.

Para abordar este tema, es importante identificar los diversos parámetros que influyen en un proceso de SLS. Uno de ellos es el asociado al polvo: su tamaño, forma, fluidez e interacción con el láser. Las formas esféricas son preferidas debido a que proporcionan una mejor densidad y fluidez al lecho de polvo. Un tamaño de partícula óptimo va de 20 a $80 \mu \mathrm{m}{ }^{[16]}$ y no es recomendable usar completamente polvos de dimensiones muy pequeñas (menores a $25 \mu \mathrm{m}$ ), debido a que pueden afectar la fluidez del lecho e incluso la interacción que este tenga con el láser.

También, otras propiedades del material, como la conductividad térmica, presión de vapor, reflectividad y punto de fusión, influyen en la interacción láser-polvo [17]. Partículas muy pequeñas son solo beneficiosas estando presentes en porciones reducidas dentro del polvo [18], [19]. Una forma irregular y un tamaño pequeño de partícula disminuyen la fluidez del lecho y, por otra parte, tamaños grandes pueden incrementar la porosidad en las capas de polvo, generando piezas con menor resistencia.

El láser es otro de los parámetros importantes en el proceso SLS; su potencia, tipo y tamaño de punto son algunos de los aspectos más relevantes, ya que pueden afectar varias características durante el proceso. La potencia y el tipo de láser varían según el material. En polímeros comúnmente se puede trabajar desde $5 \mathrm{~W}$ y altas potencias pueden incrementar los tamaños de la partícula de polvo e incluso generar baja precisión en las piezas fabricadas por posibles aumentos de tamaño [20]. Los láseres de $\mathrm{CO}_{2}$ son usados típicamente para polvos poliméricos [21]. Además, el tamaño de punto del láser debe cambiar 
en función del tipo de detalle que se requiera en las piezas, siendo una consideración importante que el tamaño de punto se relaciona con los tiempos de fabricación.

Por último, otros parámetros importantes son el espaciado y velocidad de escaneo, el espesor de capa, las estrategias de escaneo, las estrategias de construcción, el control atmosférico y las temperaturas de proceso [17]. Velocidades de escaneo altas llevan a una mayor aspereza en las piezas obtenidas y espesores de capa pequeños aumentan el tiempo de fabricación; las atmósferas no controladas pueden generar riesgos de combustión rápida y acelerar los procesos de oxidación del material [22]; en tanto que las altas temperaturas pueden modificar el tamaño medio de partículas sueltas debido a que estas se pueden sinterizar entre sí y al reciclar diversas veces el material, el tamaño continuará incrementando, lo que es considerado como no beneficioso (esto puede tener mayor efecto en aquellas partículas más cercanas a la geometría que se está construyendo, a causa de que es una zona con mayor calor) [23].

Existe una relación entre los parámetros del láser y del proceso - conocida como densidad de energía (DE) y que también puede causar efectos en las piezas obtenidas por SLS-, la cual involucra la potencia de láser, la velocidad de escaneo y el tamaño de punto. Incrementar la DE permite obtener piezas con mayor densidad [24].

De acuerdo con estimaciones del uso de materiales en 2003, el 95\% de las partes creadas por SLS fueron generadas a partir de la PA [25], entre estas la poliamida 12 (PA12), dominando el mercado debido a las facilidades que brinda en el proceso [23], [26], [27], [28]. Y a pesar de que han surgido materiales como la polieteretercetona (PEEK), los desarrollados para SLS son, en su mayoría, a base de PA 11 y 12, en combinación con otros materiales como los Nylon 11: resistente al impacto (EX) y retardante de flama (FR); los Nylon 12: relleno de aluminio (AF), reforzado con fibra de carbono (CF), con refuerzo de fibra de vidrio (GF), ligero con fibra de carbono (GSL) y reforzado con fibras minerales (HST), todos ellos producidos por Stratasys, y otros como alumide y PA2241 retardante de flama (FR), producidos por EOS [29]. Por esto, la poliamida sigue demostrando su dominio en el mercado actual de polímeros para procesos SLS.

Estudios han demostrado que para fabricar una pieza con PA12 en un proceso común de SLS solo se sinteriza del $10 \%$ al $20 \%$ del material en la cámara de construc- ción [30]. Ambos materiales (sinterizado y no sinterizado) son sometidos a cambios debido a la degradación térmica que sufren. Este deterioro ya ha sido estudiado en algunas investigaciones y su conclusión ha sido que para poder reutilizarse en otro proceso de construcción SLS es necesario mezclar el polvo reciclado de PA12 con el virgen [31], [32].

Muchas industrias dedicadas a la producción de piezas por medio del SLS optan por usar una combinación de polvos de 50/50 entre material residuo y virgen o una cantidad de $30-50 \%$ de material virgen para la mezcla en el caso de las recomendaciones emitidas por el fabricante, con el fin de evitar gran variabilidad en la calidad de las piezas [32], [33], sin embargo, otras compañías son limitadas por los requerimientos de sus clientes, optando así por usar únicamente polvo virgen de PA12.

Por lo anterior, el objetivo de esta investigación es comparar los materiales de PA12 (residuo y virgen) por medio de técnicas de microscopía, FTIR y XRD, con el fin de identificar sus diferencias morfológicas, químicas y cristalinas. El asegurar que el material extraído de un proceso de sinterizado se reutilice sin peligros, puede dar una enorme pauta para que las compañías reduzcan sus desperdicios y aprovechen sus recursos.

\section{METODOLOGÍA}

\section{OBTENCIÓN DE LOS POLVOS}

Para llevar a cabo las observaciones, las muestras de PA12 virgen $(\mathrm{PA} 12 \mathrm{~V})$ se extrajeron directamente del contenedor del fabricante Duraform (3D Systems). En el caso de la PA12 residuo (PA12R), se obtuvo de la siguiente manera: tras culminar el primer ciclo de impresión con los parámetros recomendados por el fabricante en el modo de producción estándar (PE) (Tabla 1) [34], el material restante fue sacado completamente de la máquina por medio de aspiradora, mezclándolo todo y depositándolo en recipientes cerrados. La muestra de PA12R fue extraída aleatoriamente de dicho recipiente.

TABLA 1

Parámetros Usados para el Proceso

\begin{tabular}{|l|c|}
\hline \multicolumn{1}{|c|}{ PARÁMEtro } & VALOR EN MODO PE \\
\hline Punto de ajuste del calentador de cama & $168.5^{\circ} \mathrm{C}$ \\
\hline Espesor de capa de polvo & $0.1 \mathrm{~mm}$ \\
\hline Potencia del láser & $20 \mathrm{~W}$ \\
\hline Espaciado del escaneo & $0.20 \mathrm{~mm}$ \\
\hline
\end{tabular}




\section{MICROSCOPÍA ELECTRÓNICA DE BARRIDO}

Se tomaron muestras de material PA12 en polvo para las diferentes pruebas y como técnica de análisis morfológico se usó la microscopía electrónica de barrido por medio del equipo JEOL JSM -7000F. Las muestras se analizaron sin preparación, adhiriendo una delgada capa de cinta de carbono al portamuestras donde se ubican la PA12V y la PA12R de la marca DuraForm ProX, de 3D Systems (EUA). Los análisis se realizaron aplicando aumentos de 500X con una potencia de 20 $\mathrm{kV}$ y una distancia de trabajo de $10 \mathrm{~mm}$ al equipo. Se tomaron micrografías en electrones secundarios (SEI) y se utilizó un EDS para el análisis elemental de la composición de los polvos.

\section{ESPECTROSCOPÍA POR TRANSFORMADA DE FOURIER}

Para el análisis FTIR (Fourier-Transform Infrared Spectroscopy) se utilizó el espectrofotómetro infrarrojo con transformada de Fourier (Nicolet 6700, EUA) con una configuración de 100 escaneos y $16 \mathrm{~cm}^{-1}$. Las muestras fueron analizadas en modo Reflexión Atenuada Total sin preparación previa.

\section{DIFRACCIÓN POR RAYOS X}

Para los análisis de difracción de rayos $\mathrm{X}$ se tomaron muestras de ambos materiales en polvo y el equipo usado fue el PANalytical X'pert PRO (Reino Unido). Los parámetros de la prueba utilizados fueron $2 \theta=10^{\circ}$ hasta $2 \theta=100^{\circ}$, con una velocidad de escaneo de $0.016^{\circ}$ cada 20 segundos.

\section{RESULTADOS Y DISCUSIÓN}

\section{MICROSCOPÍA ELECTRÓNICA DE BARRIDO}

La Figura 1 muestra la micrografía obtenida para la muestra de polvo PA12V con aumento de 500X en electrones secundarios y la Figura 2 expone la micrografía obtenida para la muestra de PA12R a 500X de aumento en electrones secundarios.

Las partículas del polvo de PA12V muestran una morfología amorfa, además de presentar múltiples tamaños de partícula. Igualmente, si se observa la Figura 2, correspondiente al SEM realizado de la PA12R, las partículas no siguen una geometría particular. El tamaño de partícula observado permite identificar diferencias, pues se observan incluso granos con mayores tamaños a la PA12V.

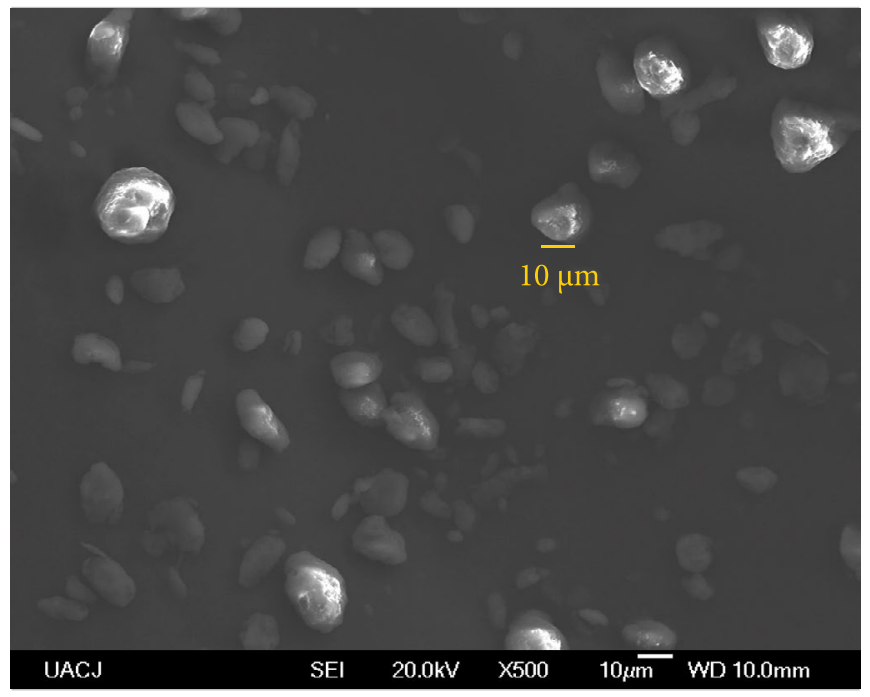

Figura 1. PA12V 500X electrones secundarios.

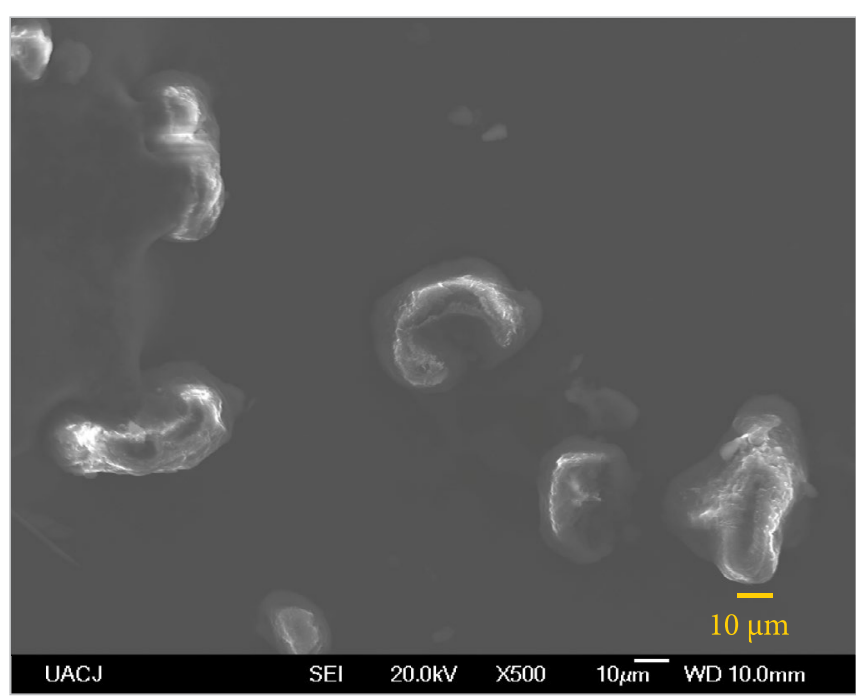

Figura 2. PA12R 500X electrones secundarios.

Para medir el tamaño de partícula se usó el software de código abierto ImageJ, haciendo mediciones aleatorias sobre las micrografías obtenidas a 500X en electrones secundarios. Considerando rangos de tamaños de partícula de 5 micras, se generó el histograma de la Figura 3, el cual muestra la distribución de concentración de tamaño de partícula para las muestras de PA12V y PA12R, y ahí se advierte que existen partículas con mayor tamaño en el polvo residuo, suposición que se había tomado inicialmente en la visualización morfológica. Para esto se planteó una comparación entre ambas calidades de polvo a partir de los SEM 
mostrados en las Figuras 2 y 3, en conjunto con los datos obtenidos del histograma.

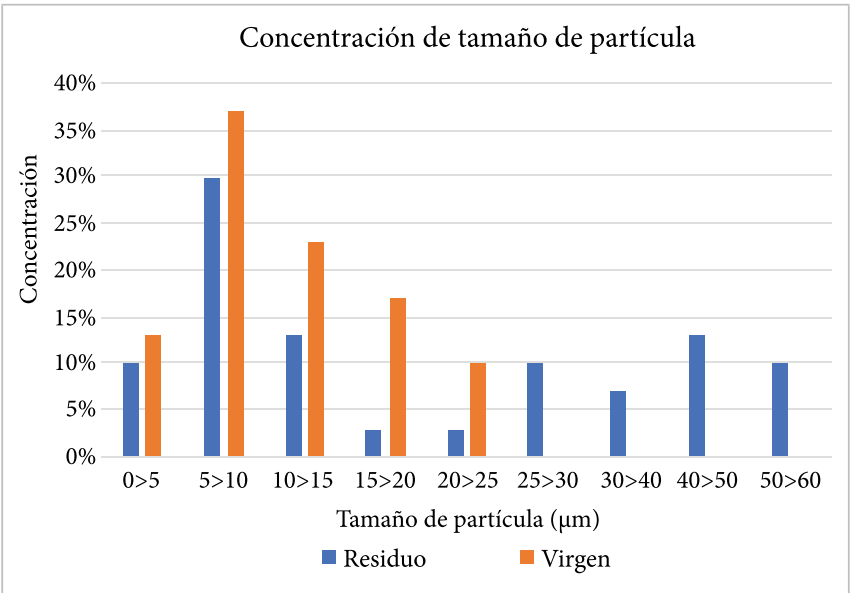

Figura 3. Concentración de tamaño de partícula para la PA12 en calidad de polvo virgen y residuo.

Para el polvo PA12V se observaron partículas con geometrías no homogéneas, algunas con aristas más pronunciadas que otras. Esto puede verificarse en la Figura 1, donde se muestran la micrografía obtenida a 500X. Las mediciones hechas presentan una media $\overline{\mathrm{x}}=10.9$ $\mu \mathrm{m}$ en el tamaño de partícula, con una desviación estándar $\mathrm{s}=5.47 \mu \mathrm{m}$ respecto de la media. La concentración de partículas recae mayormente en el rango de 5 a $10 \mu \mathrm{m}$, con un valor de $37 \%$, seguido de un $23 \%$ encontrado en tamaños de 10 a $15 \mu \mathrm{m}$ (Figura 3).

Por su parte, la PA12R mostró una morfología no homogénea, al igual que en el caso de su calidad PA12V. Sin embargo, su tamaño de partícula es mayor, con una $\overline{\mathrm{x}}=22.17 \mu \mathrm{m}$ y una $\mathrm{s}=18.28 \mu \mathrm{m}$, por lo que se contempla que el polvo cuenta con una gran variedad de tamaños de partícula. Asimismo, el mayor valor de concentración se halla en el rango de 5 a $10 \mu \mathrm{m}$ en un 30\%; $26 \%$ de forma conjunta en los rangos 10 a $15 ; 40$ a 50 y $30 \%$ en forma conjunta en los rangos 0 a 5,25 a 30 y 50 a 60 (Figura 3), lo que brinda una explicación del valor tan alto respecto a la desviación estándar obtenida.

Además, el polvo permanece a temperatura cercana al punto de fusión antes de su procesamiento y durante la extracción de piezas se presenta su proceso de enfriamiento. Otra consideración respecto a este tamaño de partícula puede deberse a que algunas de estas partículas con menor distancia al sólido no se adhieran a la pieza fabricada y queden en el restante del lecho de polvo constructivo, formando partículas con un mayor tamaño.
TABLA 2

Resultados Estadísticos De Muestras PA12V y PA12R

\begin{tabular}{|l|c|c|}
\hline \multirow{2}{*}{\multicolumn{1}{|c|}{ CaraCterística }} & \multicolumn{2}{c|}{ Muestra } \\
\cline { 2 - 3 } & PA12V & PA12R \\
\hline Número de datos & 30 & 30 \\
\hline Media $(\overline{\mathrm{x}})(\mu \mathrm{m})$ & 10.90 & 22.17 \\
\hline Desviación estándar $(\mathrm{s})(\mu \mathrm{m})$ & 5.47 & 18.28 \\
\hline Tamaño mínimo $(\mu \mathrm{m})$ & 3.17 & 2.67 \\
\hline Tamaño máximo $(\mu \mathrm{m})$ & 20.59 & 57.99 \\
\hline P10 & 4.53 & 4.39 \\
\hline P50 & 9.77 & 14.25 \\
\hline P90 & 20.22 & 43.65 \\
\hline
\end{tabular}

Estadísticamente por medio de los percentiles (Tabla 2), para la calidad de polvo virgen, el 90\% (P90) de las partículas tienen un tamaño igual o menor de $20.224 \mu \mathrm{m}$, el $50 \%$ (P50) es igual o menor a $9.77 \mu \mathrm{m}$ y el 10\% (P10) es igual o inferior a $4.53 \mu \mathrm{m}$. Por otra parte, el polvo residuo tiene un P90 de $43.65 \mu \mathrm{m}$, un P50 de $14.254 \mu \mathrm{m}$ y un P10 de $4.34 \mu \mathrm{m}$. Al comparar estos valores se observa que para un P90 existen tamaños de partícula mayores en el polvo residuo respecto al virgen y al disminuir el valor del percentil los tamaños de partícula tienden a igualar sus concentraciones de tamaño para cada percentil.

Una prueba t pareada de dos muestras se llevó a cabo con el fin de identificar si estadísticamente hay una diferencia significativa entre el tamaño de partículas de ambas calidades de polvo. Para ello se usó un nivel de confianza del 95\% ( $\mathrm{P}=0.05)$. Los resultados obtenidos arrojan un valor $\mathrm{P}$ de 0.003 , menor que el nivel de significancia establecido. Por tal razón, la hipótesis nula planteada dice que no existe diferencia significativa entre las medias de ambas muestras y, por tanto, es rechazada. Lo anterior indica que estadísticamente sí existe diferencia entre los tamaños de partícula de ambos polvos (Figura 4).

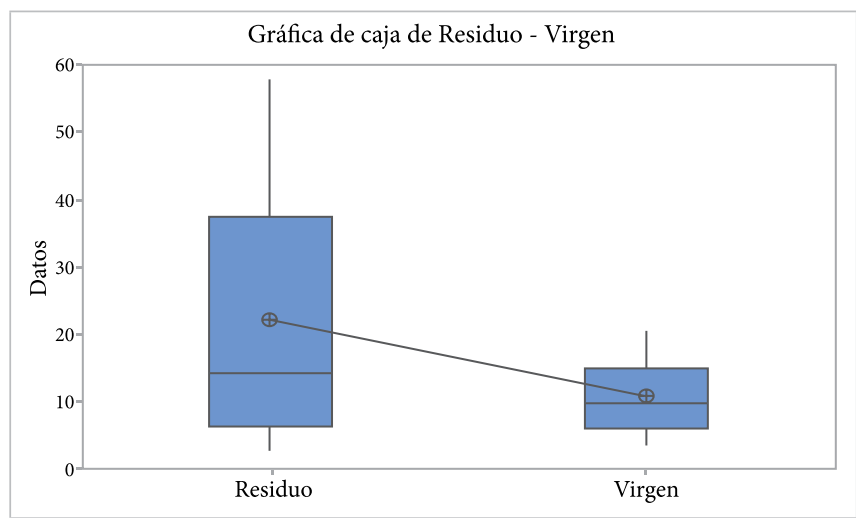

Figura 4. Gráfica de caja para prueba t a tamaños de partícula a calidades de polvo. 


\section{ESPECTROSCOPIA DE RAYOS X DE ENERGÍA DISPERSA (EDS)}

Para un análisis de composición se realizaron EDS a las muestras de polvo en ambas calidades, con la finalidad de observar los elementos químicos que componen la muestra y observar qué diferencias se pueden apreciar entre ambas calidades de material. Las Figura 5 presenta los espectros (a) PA12R y (b) PA12V.

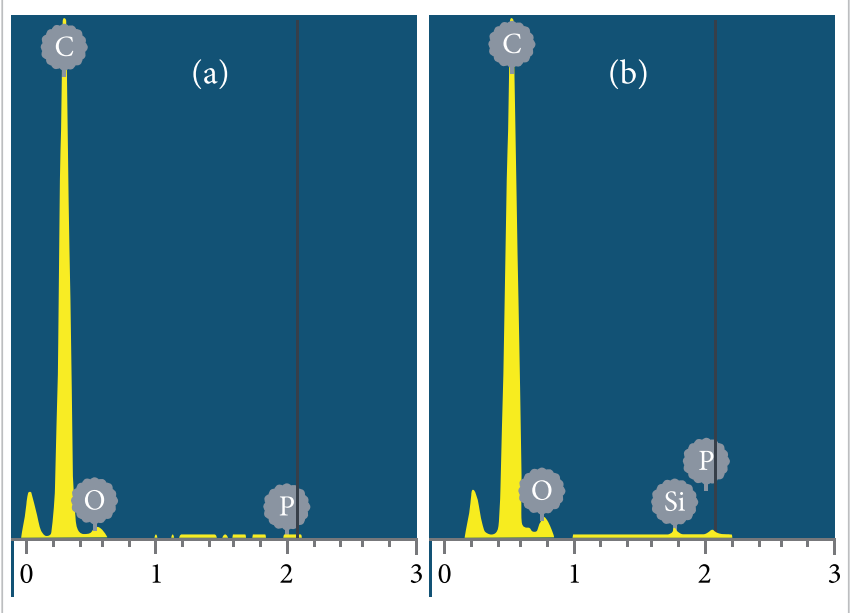

Figura 5. Espectro de energía dispersa: (a) PA12R y (b) PA12V.

Para ambos espectros se observan picos de composición similares. En ambos casos, el más alto pertenece al carbono y otros elementos, como el oxígeno y fósforo son vistos igualmente, sin embargo, se nota la aparición de un pequeño pico de silicio, posiblemente perteneciente a un pico de fluorescencia interna de silicio no originado en la muestra. La Tabla 3 permite observar los valores en porcentajes de peso de cada elemento en ambos espectros mostrados.

TABLA 3

VAlores de Composición POR Elemento En Espectros de ENERGÍA DISPERSA

\begin{tabular}{|c|c|c|c|}
\hline Muestra & Elemento & Peso (\%) & Atómico (\%) \\
\hline PA12R & $\mathrm{C}$ & 90.22 & 92.39 \\
\cline { 2 - 4 } & $\mathrm{O}$ & 9.77 & 7.53 \\
\cline { 2 - 4 } & $\mathrm{P}$ & 0.21 & 0.08 \\
\cline { 2 - 4 } & Total & 100 & 100 \\
\hline PA12V & $\mathrm{C}$ & 85.21 & 88.65 \\
\cline { 2 - 4 } & $\mathrm{O}$ & 14.21 & 11.1 \\
\cline { 2 - 4 } & $\mathrm{Si}$ & 0.33 & 0.15 \\
\cline { 2 - 4 } & $\mathrm{P}$ & 0.25 & 0.1 \\
\cline { 2 - 4 } & Total & 100 & 100 \\
\hline
\end{tabular}

\section{ESPECTROSCOPIA POR TRANSFORMADA} DE FOURIER (FTIR)

En la Figura 6 se muestran los espectros encontrados del análisis FTIR para los polvos PA12V y PA12R. Las bandas de $3295 \mathrm{~cm}^{-1}$ y $1555 \mathrm{~cm}^{-1}$ son valores correspondientes a los grupos de $-\mathrm{NH}$ de vibraciones de estiramiento y de flexión respectivamente, las magnitudes encontradas entre $2800 \mathrm{~cm}^{-1}$ y $3000 \mathrm{~cm}^{-1}$ se atribuyen a los grupos de $-\mathrm{OH}$ y la banda en $1640 \mathrm{~cm}^{-1}$ se relacionan al grupo $-\mathrm{C}=\mathrm{O}{ }^{[35]}$. Ambas calidades de polvo presentan espectros con bandas muy similares, indicando los mismos grupos funcionales en ambas muestras, lo que permite inferir que el material residuo no presenta una variación química respecto al polvo virgen.

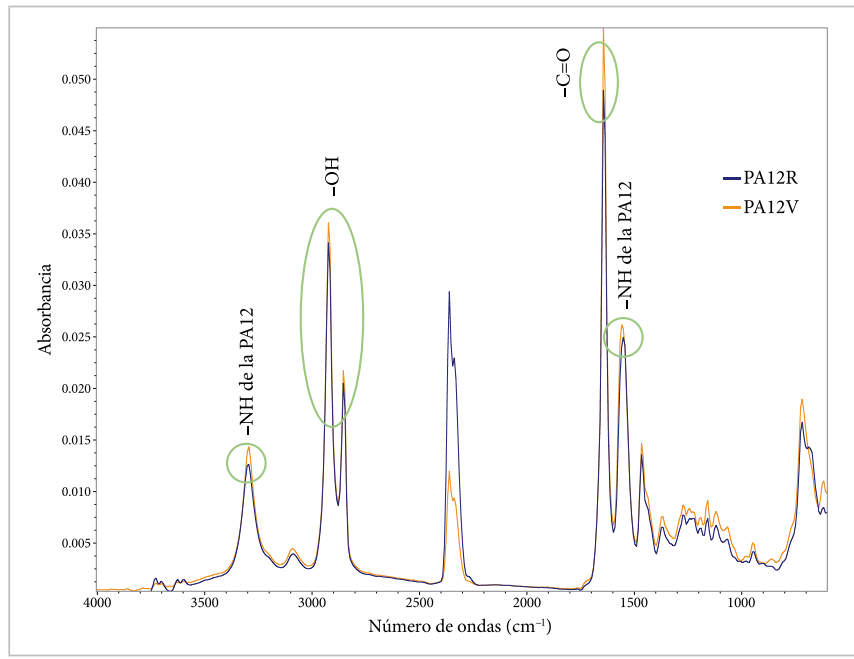

Figura 6. Comparación de espectros infrarrojos por transformada de Fourier de polvos PA12V y PA12R. Fuente: los autores.

\section{DIFRACCIÓN POR RAYOS X (XRD)}

Los espectros de la Figura 7 muestran los picos obtenidos durante la prueba XRD para los polvos PA12V y PA12R. En ambas calidades se identifican dos picos predominantes: en $2 \theta=21.16^{\circ}$ y $22.18^{\circ}$ para PA12V y $2 \theta=21.35^{\circ}$ y $22.66^{\circ}$ para el PA12R. El pico alrededor de $2 \theta=21^{\circ}$ en ambas muestras se atribuye el plano (001) característico de la forma $\gamma$, siendo esta la estructura cristalina más común en el Nylon 12 [35]. Sin embargo, la aparición de estos picos duales de alrededor de $21^{\circ} \mathrm{y}$ $22^{\circ}$ se asocian típicamente en las poliamidas a una falta de coincidencia entre los espacios intercadena e interplanos [36]. Además de los picos duales, también se muestra otra leve difracción en $2 \theta=11.16^{\circ}$, al cual se le podría atribuir el plano (040) [37]. Los patrones mostra- 
dos en ambos materiales son muy similares, lo que indica que en ambas calidades no se encuentran diferencias significativas respecto a sus planos de difracción.

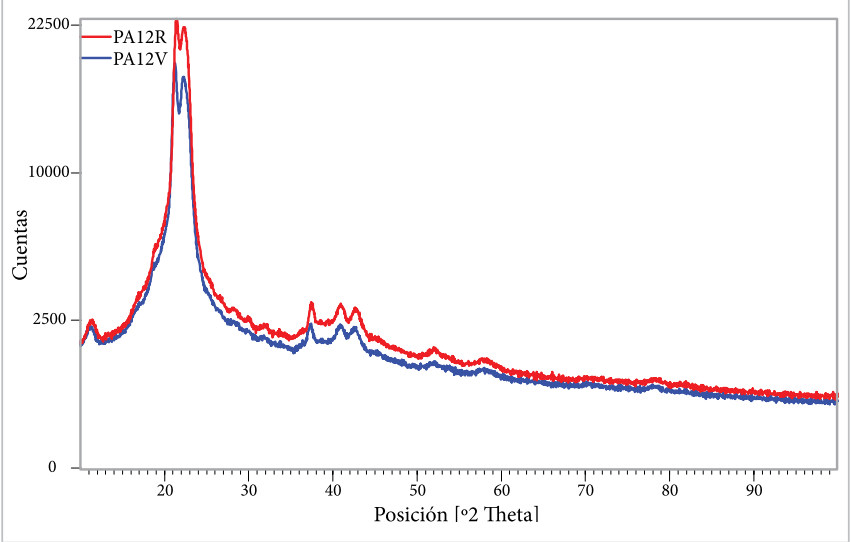

Figura 7. Patrones de difracción de rayos $\mathrm{X}$ de poliamida 12 (PA12). Fuente: los autores.

El tamaño de partícula obtenido para los polvos tratados indica que existen diferencias en este aspecto para ambas calidades de polvo tratadas, sin embargo, esto difiere de los resultados obtenidos por Pham et al. [31] y Dadbakhsh et al. [26], quienes muestran que el tamaño de partícula y su morfología no varían en ambas calidades de polvo tras un envejecimiento. Según se considera en ambos estudios, estas diferencias pueden deberse a varios factores, como el acondicionamiento de muestras, las marcas comerciales de material que se ha usado para la recolección de muestras, la zona de extracción de la muestra y la realización de pruebas en cada investigación. En este caso, en ambas investigaciones se usaron polvos con base PA12, sin embargo, los proveedores son diferentes a los empleados en la presente investigación, lo que puede explicar las variaciones. Asimismo, la extracción de la muestra en este caso se tomó de forma aleatoria, pues todo el polvo residuo fue extraído y depositado en un mismo recipiente.

También para el polvo PA12R se han conseguido mayores tamaños de partícula respecto al virgen, sin embargo, observando lo planteado por Schmid et al. [16], gran parte de los tamaños obtenidos para el residuo de PA12R en la presente investigación se encuentran en el rango del tamaño de partícula que puede usarse dentro de los procesos SLS.

$\mathrm{El}$ análisis por difracción de rayos $\mathrm{X}$ evidenció que las muestras PA12V y PA12R no presentan diferencias en las fases cristalinas y según los resultados mostrados por
Ma et al. [37], el material de estudio en ambas calidades se asociaría a una fase $\alpha$ de la PA12 debido a la aparición de los picos duales observados. Sin embargo, Bain et al. [36], indican que dichos picos duales en polvos de PA12 en su estado virgen ( $\sin$ sinterizar) pueden deberse a fases cristalinas en desequilibrio, puntualizando que el material observado Duraform ProX PA de 3D Systems tiene una fase semicristalina entre $\alpha$ y $\gamma$.

\section{CONCLUSIONES}

El estudio de materiales tiene gran importancia para el desarrollo tecnológico y más aún cuando los materiales de interés son generados a partir de residuos de procesos de fabricación. Esto permite observar los cambios del material y concluir si es posible reutilizarlo en el mismo proceso e incluso identificar otras posibles aplicaciones.

Por medio de la prueba t pareada se observó que las medias del tamaño de partícula de los polvos de PA12V y PA12R presentan una diferencia significativa. Por lo tanto, se puede afirmar que existen cambios en este parámetro del material tras un ciclo de proceso. Esto pudiera cambiar la composición estructural de las piezas impresas en los equipos SLS si no son mezcladas como lo recomiendan los fabricantes y diversos autores.

Al tener partículas más grandes, las capas generadas se formarán con una mayor porosidad debido al incremento de espacios en el empaquetamiento de partículas, llevando así a la generación de piezas menos resistentes. Una mezcla de materiales permite disminuir el tamaño de dichos espacios y por ende disminuir la porosidad de las capas depositadas.

Por medio de procesos simples, como el de tamizado, se podría llegar a mejorar este aspecto en la PA12R al filtrar los tamaños de partícula más grandes que se encuentren por encima de lo recomendado o que sobrepasen el espesor de capa que se desea usar, permitiendo aumentar la calidad en las piezas fabricadas e incluso incrementar la cantidad de PA12R a reusar en el proceso.

La evidencia proporcionada por el análisis de FTIR y XRD arrojan que la calidad de ambos polvos es similar y no se distingue ningún efecto del proceso previo en el PA12R en las propiedades químicas y cristalinas de las sustancias, lo que favorecería el uso y reciclaje del PA12R en procesos de SLS. Esto significaría resultados 
prometedores en el reúso de la sustancia para la elaboración de partes impresas, con ahorros en material y mayor compromiso con el ambiente.

Por último, en el transcurso de la presente investigación se han identificado opciones de investigación futura relacionadas con la comparación de propiedades mecánicas, químicas y térmicas de piezas elaboradas a partir de PA12V y PA12R tras ciclos cortos de uso, así como para identificar si existen variaciones en dichas propiedades. También, se sugiere desarrollar estrategias novedosas para la disminución de desperdicios en procesos de sinterizado láser, las formas de recuperación y la reimplementación en otros procesos de manufactura aditiva, así como estudios de diversidad de polvos como el PEEK y las derivaciones de materiales a partir de la PA tras condiciones de envejecimiento a ciclos cortos y largos para observar sus efectos en las piezas generadas.

\section{REFERENCIAS}

[1] PlasticsEurope, "Plastics - the Facts 2016. An analysis of European plastics production, demand and waste data", Plastics Europe. Bruselas, Bélgica, 2016. [En línea]. Disponible: https://www.plasticseurope.org/ application/files/4315/1310/4805/plastic-the-fact-2016. pdf. [Consultado: ag. 4, 2020].

[2] L. W. McKeen, "Polyamides (Nylons)", en The Effect of UV Light and Weather on Plastics and Elastomers, $4 .^{\mathrm{a}} \mathrm{ed}$. L. W. McKeen, Ed. Kidlington, Oxford: William Andrew Publishing, 2019, cap. 7, pp. 185-222.

[3] G. Wypych, "PA-12 polyamide-12", en Handbook of Polymers, 2. a ed. G. Wypych, Ed. Toronto, Ontario: ChemTec Publishing, 2016, pp. 246-250.

[4] R. Singh y S. Singh, "Additive Manufacturing: An Overview", Reference Module in Materials Science and Materials Engineering, 2017, doi: 10.1016/b978-0-12-8035818.04165-5.

[5] C. R. Deckard, "Method and apparatus for producing parts by selective sintering”, U.S. Patent 4863538 , sept. $5,1989$.

[6] S. C. Ligon, R. Liska, J. Stampfl, M. Gurr y R. Mülhaupt, "Polymers for 3D Printing and Customized Additive Manufacturing", Chem. Rev., vol. 117, no. 15, pp. 1021210290, ag. 2017, doi: 10.1021/acs.chemrev.7b00074.
[7] C. Cai et al., "Comparative study on 3D printing of polyamide 12 by selective laser sintering and multi jet fusion", J. Mater. Process. Technol., vol. 288, feb. 2021, doi: 10.1016/j.jmatprotec.2020.116882.

[8] J. W. Halloran et al., "Photopolymerization of powder suspensions for shaping ceramics", J. Eur. Ceram. Soc., vol. 31, no. 14, pp. 2613-2619, nov., 2011, doi: 10.1016/j. jeurceramsoc.2010.12.003.

[9] H.-H. Tang, M.-L. Chiu y H.-C. Yen, "Slurry-based selective laser sintering of polymer-coated ceramic powders to fabricate high strength alumina parts", J. Eur. Ceram. Soc., vol. 31, no. 8, pp. 1383-1388, jul., 2011, doi: 10.1016/j.jeurceramsoc.2011.02.020.

[10] G. V. Salmoria, R. A. Paggi, A. Lago y V. E. Beal, "Microstructural and mechanical characterization of PA12/ MWCNTs nanocomposite manufactured by selective laser sintering", Polym. Test., vol. 30, no. 6, pp. 611-615, sept., 2011, doi: 10.1016/j.polymertesting.2011.04.007.

[11] K. V. Wong y A. Hernandez, "A Review of Additive Manufacturing", ISRN Mechanical Engineering, vol. 2012, Ag. 2012, doi: 10.5402/2012/208760.

[12] R. Singh et al., "Powder bed fusion process in additive manufacturing: An overview", Materials Today: Proceedings, vol. 26, parte 2, pp. 3058-3070, 2020, doi: 10.1016/j. matpr.2020.02.635.

[13] “3D SYTEMS”, 3dsystems.com, 2013. [En línea]. Disponible: https://www.3dsystems.com/. [Consultado: oct. 13, 2020].

[14] "Professional SLS 3D Printers For Research, Prototyping And Production", Sintratec.com, 2020. [En línea]. Disponible: https://sintratec.com/. [Consultado: oct. 13, 2020].

[15] "Additive Manufacturing solutions \& industrial 3D printer by EOS”, EOS.info, 2020. [En línea]. Disponible: https://www.eos.info/en [Consultado: oct. 13, 2020].

[16] M. Schmid, A. Amado y K. Wegener, "Materials perspective of polymers for additive manufacturing with selective laser sintering", J. Mater. Res., vol. 29, no. 17, pp. 1824-1832, sept. 2014, doi: 10.1557/jmr.2014.138.

[17] S. Kumar, "Selective Laser Sintering/Melting", en Comprehensive Materials Processing, vol. 10, S. Hashmi, G. 
Ferreira Batalha, C. J. Van Tyne y B. Yilbas, Eds. Elsevier, 2014, pp. 93-134.

[18] Y. Shi, Z. Li, H. Sun, S. Huang y F. Zeng, "Effect of the properties of the polymer materials on the quality of selective laser sintering parts", P I MECH ENG L-J MAT, vol. 218, no. 3, pp. 247-252, jul. 2004, doi: $10.1177 / 146442070421800308$.

[19] S. Ziegelmeier, P. Christou, F. Wölleck, C. Tuck, R. Goodridge, R. Hague, E. Krampe y E. Wintermantel, "An experimental study into the effects of bulk and flow behaviour of laser sintering polymer powders on resulting part properties", J. Mater. Process. Technol., vol. 215, pp. 239-250, En. 2015, doi: 10.1016/j.jmatprotec.2014.07.029.

[20] 3D Systems, "Duraform ${ }^{\circledR}$ ProX $^{\mathrm{TM}} \mathrm{PA}$ : Guía de Materiales", Infocenter.3dsystems.com, Circle Rock Hill, SC, 2018. [En línea]. Disponible: http://infocenter.3dsystems. com/product-library/sites/default/files/ProX_6100/ Customer/Spanish/134015-00_DuraForm_ProX_PA_ Material Guide_Rev_B_es-xn.pdf. [Consultado: feb. 26, 2021].

[21] M. M. Savalani, L. Hao y R. A. Harris, "Evaluation of $\mathrm{CO}_{2}$ and Nd:YAG Lasers for the Selective Laser Sintering of HAPEX ${ }^{\circledR ”,}$ P I MECH ENG B-J ENG, vol. 220, no. 2, pp. 171-182, 2006, doi: 10.1243/095440505X32986.

[22] S. Bajric, "Selective Laser Sintering of PA2200: Effects of print parameters on density, accuracy, and surface roughness", U.S. Patent LA-UR-17-24716, jun., 12, 2017, doi: 10.2172/1363740.

[23] I. Gibson, D. Rosen y B. Stucker, Additive Manufacturing Technologies: 3D Printing, Rapid Prototyping, and Direct Digital Manufacturing. Nueva York: Springer, 2014.

[24] B. Caulfield, P. E. McHugh y S. Lohfeld, "Dependence of mechanical properties of polyamide components on build parameters in the SLS process", J. Mater. Process. Technol., vol. 182, no. 1-3, pp. 477-488, feb. 2007, doi: 10.1016/j.jmatprotec.2006.09.007.

[25] J. P. Kruth, X. Wang, T. Laoui y L. Froyen, "Lasers and materials in selective laser sintering", Assembly Automation, vol. 23, no. 4, pp. 357-371, en. 2003. doi: $10.1108 / 01445150310698652$.
[26] S. Dadbakhsh, L. Verbelen, O. Verkinderen, D. Strobbe, P. Van Puyvelde y J.-P. Kruth, "Effect of PA12 powder reuse on coalescence behaviour and microstructure of SLS parts", European Polymer Journal, vol. 92. pp. 250262, 2017, doi: 10.1016/j.eurpolymj.2017.05.014.

[27] M. Schmida, A. Amadoa y K. Wegenerb, "Polymer powders for selective laser sintering (SLS)", AIP Conference Proceedings, vol. 1664, no. 1, may. 22, 2015, doi: $10.1063 / 1.4918516$.

[28] D. Drummer, K. Wudy y M. Drexler, "Selective laser melting of polyamide 12: a holistic approach for modeling of the aging behaviour", en Proceedings 5th International Conference on Additive Technologies - iCAT2014, Ljubljana, 2014, pp. 216-221.

[29] H. Wu, W. P. Fahy, S. Kim, H. Kim, N. Zhao. L. Pilato, A. Kafi, S. Bateman y J. H. Koo, "Recent developments in polymers/polymer nanocomposites for additive manufacturing", Prog. Mater Sci., vol. 111, jun. 2020, doi: 10.1016/j.pmatsci.2020.100638.

[30] K. Dotchev y W. Yusoff, "Recycling of polyamide 12 based powders in the laser sintering process", Rapid Prototyping Journal, vol. 15, no. 3, pp. 192-203, en. 2009, doi: 10.1108/13552540910960299.

[31] D. T. Pham, K. D. Dotchev y W. A. Y. Yusoff, "Deterioration of polyamide powder properties in the laser sintering process", Proc Inst Mech Eng Part C, vol. 222, no. 11, pp. 2163-2176, nov. 5, 2008, doi: 10.1243/09544062JMES839.

[32] A. C. Lopes, Á. M. Sampaio, C. S. Silva y A. J. Pontes, "Prediction of SLS parts properties using reprocessing powder", Rapid Prototyping Journal, vol. 39, p. 88, feb. 2021, doi: 10.1108/RPJ-04-2020-0076.

[33] S. Kumar y A. Czekanski, "Development of filaments using selective laser sintering waste powder", J. Clean. Prod., vol. 165, pp. 1188-1196, nov. 2017, doi: 10.1016/j. jclepro.2017.07.202.

[34] 3D Systems, “3D Systems Legacy Printers”, 3D Systems, Infocenter.3dsystems.com, 2020. [En línea]. Disponible: http://infocenter.3dsystems.com/product-ibrary/ system/files/legacy/3DSCentral-ProductionPrinters/ ProX500/Customer/Manuals/2484_CIB1601-Building 
withDuraFormProXPAEngineeredThermoplastic.pdf. [Consultado: feb. 28, 2021].

[35] R. Rafiq, D. Cai, J. Jin y M. Song, "Increasing the toughness of nylon 12 by the incorporation of functionalized graphene", Carbon, vol. 48, no. 15. pp. 4309-4314, dic. 2010, doi: 10.1016/j.carbon.2010.07.043.

[36] E. D. Bain, E. J. Garboczi, J. E. Seppala, T. C. Parker y K. B. Migler, "AMB2018-04: Benchmark Physical Property
Measurements for Powder Bed Fusion Additive Manufacturing of Polyamide 12", Integr. Mater. Manuf. Innov., vol. 8, no. 3. pp. 335-361, 2019, doi: 10.1007/s40192019-00146-3.

[37] N. Ma, W. Liu, L. Ma, S. He, H. Liu, Z. Zhang, A. Sun, M. Huang y Ch. Zhu, "Crystal transition and thermal behavior of Nylon 12", e-Polymers, vol. 20, no. 1. pp. 346352, jun. 2020, doi: 10.1515/epoly-2020-0039. 